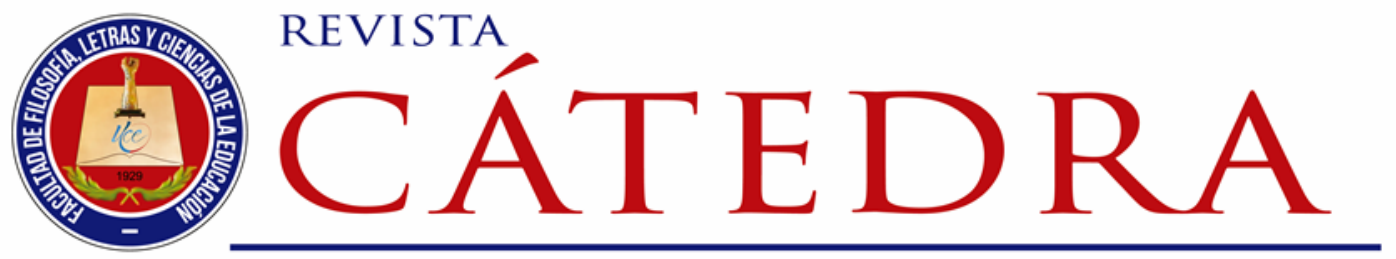

\title{
Análisis lingüístico femenino en el siglo XXI a través del discurso de Cynthia Viteri
}

\section{Female linguistic analysis in the 21st Century: the speech of Cynthia Viteri}

\author{
Miguel Román-Jaramillo \\ Universidad Central del Ecuador, Quito, Ecuador \\ maromanj@uce.edu.ec \\ https://orcid.org/0000-0002-9789-9753
}

Magali Arévalo-Arteta

Universidad Central del Ecuador, Quito, Ecuador

mjarevalo@uce.edu.ec

https://orcid.org/0000-0002-1314-8413

(Recibido: 11/11/2019; Aceptado: 18/11/2019; Versión final recibida: 08/01/2020)

Cita del artículo: Roman-Jaramillo, M., Arévalo-Arteta, M., (2020). Análisis lingüístico femenino en el siglo XXI a través del discurso de Cynthia Viteri. Revista Cátedra, 3(1), 94115.

\section{Resumen}

El propósito de este artículo es presentar de una manera general, mediante ejemplos prácticos, un análisis discursivo. Como sujeto de análisis se consideró a la Dra. Cynthia Viteri, por ser actualmente una de las mujeres más destacas en la política ecuatoriana y la máxima autoridad de la ciudad de Guayaquil, Ecuador. La antropología lingüística, la sociolingüística, la nueva dialectología y lo que en el ámbito anglosajón se denomina la "lingüística feminista" llevan varias décadas aportando datos que muestran cómo hombres y mujeres se construyen y se manifiestan de forma diferente en cuanto a la manera de utilizar las lenguas. El presente trabajo de investigación tiene un enfoque descriptivo, y se desarrolló mediante la observación continua de videos y conferencias emitidos por la actual alcaldesa de Guayaquil, Dra. Cynthia Viteri Jiménez, mismos que se reproducen en el canal de Youtube y corresponden a los meses entre enero y noviembre del 2019. El análisis lingüístico demuestra la combinación y conexión de expresiones que exteriorizan los actos comunicativos. Se hace mayor hincapié en formas lingüísticas como el léxico, la referencia, la coherencia (argumentación), la interacción de semántica y sintaxis (modalidad, aspectos, 
tiempo, negación, modificación adverbial, etc.), así como también en las manifestaciones del lenguaje corporal del actor del discurso y además se puede observar que las diferencias de género son un hecho fundamental de la vida humana y no es sorprendente encontrarlas reflejadas en el lenguaje; por lo tanto, la atención al uso del lenguaje en el contexto social es muy importante.

\section{Palabras clave}

Análisis, habla, lenguaje corporal, lenguaje femenino, lingüística.

\section{Abstract}

The aim of this article is to present through practical examples a discourse analysis of Dr. Cynthia Viteri, who was considered as the subject of this analysis because she is actually one of the most prominent women in Ecuadorian politics and the highest authority of the city of Guayaquil, Ecuador. Linguistic anthropology, sociolinguistics, the new dialectology and what in the Anglo-Saxon field is called "feminist linguistics" have been providing data for decades to show, in terms of how to use the languages, the way men and women construct and manifest themselves differently. This research work has a descriptive approach, and it was developed through the continuous observation of videos and conferences issued by the current mayor of Guayaquil, Dr. Cynthia Viteri Jiménez, which are reproduced through the YouTube channel and correspond to the months between January and November 2019. The linguistic analysis shows the combination and connection of expressions that externalize the communicative acts. Greater emphasis is placed on linguistic forms such as lexicon, reference, coherence (argumentation), the interaction of semantics and syntax (modality, aspects, time, negation, modification adverbial, etc.), as well as the speaker's body language manifestations. Although, it is possible to observe that the gender differences are a fundamental fact of human life and it is not surprising to find them reflected in language; therefore, attention to the use of language in the social context is very important.

\section{Keywords}

Analysis, spech, body language, female language, linguistics.

\section{Introducción}

Cada vez son más abundantes los estudios que abordan las relaciones entre los usos lingüísticos y las diferencias sexuales. La antropología lingüística, la sociolingüística, la nueva dialectología y lo que en el ámbito anglosajón se denomina la "lingüística feminista" llevan varias décadas aportando datos que muestran cómo hombres y mujeres se construyen y se manifiestan de forma diferente en cuanto a la manera de utilizar las lenguas. Asimismo, "se están desvelando los usos sexistas de las lenguas y proponiendo formas de uso que permitan nombrar a las mujeres” (Tusón, 2016, pág. 138).

El presente trabajo de investigación tiene un enfoque descriptivo, y se desarrolló mediante la observación continua de videos y conferencias emitidos por la actual alcaldesa de Guayaquil Dra. Cynthia Viteri Jiménez, mismos que se reproducen en el canal de Youtube y corresponden al período comprendido entre los meses de enero y noviembre del 2019. Los principales ejes que han promovido la investigación en torno de la diversidad lingüística ligada a la diferencia sexual han sido las diferencias en la adquisición lingüística según el sexo; las formas de transmisión cultural y, especialmente, la adquisición y el desarrollo de la competencia comunicativa en niños y niñas, chicos y chicas. Desde la perspectiva lingüístico-discursiva, los primeros trabajos sobre la diversidad lingüística y la diferencia 
sexual que han tenido un gran impacto y se han convertido en referencia obligada son los de Lakoff (1975) y Tannen (1994). De igual forma, para conocer la opinión de lingüistas y gramáticos en lo que se refiere al habla de las mujeres, véase el capítulo tercero de la obra de Lozano Domingo (1995), así como el libro de Yaguello (1978). También, desde la perspectiva del análisis crítico del discurso, véanse los trabajos que aparecen en la obra de Wodak (1997).

Este trabajo de investigación está conformado por cuatro partes: la primera corresponde a la recopilación de los videos de Cynthia Viteri, desde el mes de enero hasta noviembre del 2019; como segunda parte, se realizó la observación de los videos enfocada en los aspectos verbales y no verbales; como tercera parte, se analizó detalladamente los aspectos lingüísticos del discurso y finalmente, se registraron los ejemplos correspondientes a los temas relacionados con el análisis discursivo particular dentro de los aspectos lingüísticos femeninos.

\section{Lenguaje femenino del silgo XXI}

Mucho se habla de los avances de la postura femenina que irrumpió en la década de los setenta y se fortaleció en los ochenta, sin embargo, en lo referente a la mujer, "desde tiempos remotos y como consecuencia de sus características y posibilidades biológicas y naturales, se la preparaba para cuidar a los hijos y educarlos amorosamente, enseñándoles la posibilidad de ser expresivos y mostrar emociones ante las distintas situaciones de vida" (Moreno, 2016, pág. 1). Esto significa que la mujer era educada para permanecer en casa, formar un buen hogar, ser una buena ama de casa y esposa ejemplar. El aprendizaje de la mujer era en el ámbito materia de las labores domésticas, atención y cuidados para su esposo y sus hijos. Es decir, el papel de esposa era considerado como el más importante para desempeñarse en un medio social.

Además, ciertos autores señalan que "el siglo XX ha sido el del descubrimiento de las mujeres, el de su revolución y ahora en el siglo XXI será el siglo de las mujeres" (Arnedo, et al., 2006, pág. 2). A pesar de que es cierto que a la mujer le queda aún mucho camino por recorrer para pasar de los derechos a los hechos cabe reconocer que en los últimos estudios sobre productividad recomiendan la promoción de las mujeres para la dirección empresarial. Parece que las habilidades femeninas son más rentables para los modernos sistemas productivos de trabajo en red, en grupo, solidario y abierto.

Como ejemplos de los avances de la mujer en la política del siglo XXI, podemos citar lo siguiente:

El actual Gobierno noruego obliga que haya por lo menos dos mujeres en todos los consejos de dirección, este siglo parece estar dando paso a las mujeres en el mundo, tan cerrada y tradicionalmente masculino, especialmente en el mundo de la política, así por ejemplo al terminar el primer lustro de este siglo, había en el mundo cinco mujeres presidentas en diferentes países: Irlanda, Letonia, Finlandia, Filipinas y Sri Lanka; y cuatro primeras ministras: Angela Merkel, recién elegida canciller de Alemania, Helen Elizabeth Clark en Nueva Zelanda, Khaleda Zia en Bangladesh, y Luisa Diogo en Mozambique. Europa, Oceanía, Asia y África, faltaba América y, al empezar el año 2006, Michelle Bachelet fue elegida presidenta de Chile. A los pocos días, Ellen Johnson Sirleaf era aclamada presidenta de Liberia. Además, cabe recalcar que han llegado a los más altos niveles de poder político cuatro mujeres en Europa, tres en 
Asia, dos en África, una en América y una en Oceanía (Arnedo, et al., 2006, pag. 3).

Como se puede evidenciar la figura femenina ha ganado prestigio en el siglo XXI, especialmente en al ámbito político y esto se puede verificar en su expresión corporal, la misma que no es tan diferente del lenguaje corporal de los hombres. La mayoría usan las expresiones faciales similares $\mathrm{y}$, en su mayor parte, el mismo lenguaje corporal para expresar cómo se sienten, cómo atraen el uno al otro, cómo buscan atención, y cuáles son sus signos de ansiedad. Las características lingüísticas propias femeninas, tales como: expresión corporal, expresión facial, morfosintaxis, léxico, proxemia, prosodia han permitido que la mujer gane terreno en todos los espacios y más reconocido en el ámbito político.

\section{Materiales y métodos}

El presente trabajo de investigación tiene un enfoque descriptivo, y se desarrolló mediante la observación continua de videos y conferencias emitidos por la actual alcaldesa de Guayaquil, Dra. Cynthia Viteri Jiménez, mismos que son transmitidos por el canal de Youtube y corresponden al período comprendido entre los meses de enero y noviembre del 2019. Actualmente, Cynthia Viteri Jiménez tiene una audiencia muy diversa, lo que la convierte en un personaje interesante para la elaboración de un artículo científico.

El procedimiento para realizar este trabajo de investigación consistió en seguir una serie de pasos, los cuales son:

1. recopilación de los videos de Cynthia Viteri, lo cual implicó descargar los videos del canal de Youtube, correspondientes a los meses desde enero a noviembre del 2019 para poder separarlos por cada uno;

2. seguidamente, se realizó la observación de todos los videos recopilados, enfocada en los aspectos verbales y no verbales desde la perspectiva de la pragmática;

3. a continuación, se analizó detallada y detenidamente los aspectos lingüísticos de los discursos: el lenguaje corporal, implicaturas, presuposiciones, actos del habla, interacción, cooperación, rapport y características lingüísticas femeninas;

4. y finalmente, después de un largo proceso de observación y análisis de todos los videos recolectados se procedió a registrar los ejemplos correspondientes a los temas relacionados con el análisis discursivo particular dentro de los aspectos lingüísticos femeninos.

Para poder realizar este proceso de investigación se utilizaron los siguientes videos:

https://www.youtube.com/watch?v=LrpIEnRs7NY; Tema: Dra. Cynthia Viteri - Radio Élite. https://www.youtube.com/watch?v=RXUL05XIj6s;Tema: Entrevista a la alcaldesa -RTS. https://www.youtube.com/watch?v=Z7e4mA8XUdg; Tema: La propuesta de Cynthia Viteri para la Alcaldía de Guayaquil. https://www.youtube.com/watch?v=3DUNxbzi-oU; Tema: Cynthia Viteri critica medidas económicas del Gobierno. https://www.youtube.com/watch? v=vPJQKA5rJMg, Tema: Cynthia Viteri - enlace Radio Centro. https://www.youtube.com/watch?v=nCKEesewxfk, Contexto: ¡Con Guayaquil no se juega!

Figura 1. Opción "Conservar solo texto" en las "Opciones de pegado" 


\section{Análisis del discurso}

Después del proceso de observación, se escogieron diferentes ejemplos tomados de los videos de Cynthia Viteri Jiménez publicados en Youtube, tal cual fueron pronunciados en sus conferencias, sin alteración alguna; tomando en cuenta su postura femenina en el ámbito político. Los ejemplos que se citan a continuación están clasificados de acuerdo a los temas analizados y explicados anteriormente.

\subsection{Deixis}

\subsubsection{Personal}

Ejemplo $01 \quad$... esto es una tarea de nunca acabar Luisa, mi compromiso no sé no solamente será continuar en la desintoxicación y seguir firmando los convenios que nos permitan salvar vidas ...

Ejemplo $02 \quad \ldots$ recién llegamos al acuerdo de la semana pasada les diré el nombre del médico cabe destacar que los médicos en algunas de estas operaciones no cobran nada nosotros ponemos hospital anestesista recuperación ponemos todos nosotros ponemos hospital anestesista recuperación ponemos todo ...

Contexto: Entrevista a la alcaldesa -RTS ${ }^{1}$

\subsubsection{Espacial}

Ejemplo 03 Guayaquil traerá los mejores equipos para este nuevo centro de rehabilitación física que además podrá atender a los niños con parálisis cerebral para este nuevo centro de rehabilitación física Contexto: Entrevista a la alcaldesa -RTS ${ }^{2}$

Ejemplo $04 \quad \ldots$ ahora podemos ver con claridad cuál es el verdadero impacto de las medidas económicas adoptadas por este gobierno... Contexto: Cynthia Viteri critica medidas económicas del Gobierno $^{3}$

\subsubsection{Temporal}

\begin{tabular}{ll}
\hline Ejemplo 05 & $\begin{array}{l}\text { Ayer acabamos de dar a nuestros niños de la Escuela "4 de enero", } \\
\text { niños no videntes, un aparatito hecho por Walt ... }\end{array}$ \\
\hline Contexto: Cynthia Viteri - enlace radio centro \\
\hline Ejemplo 06 & $\begin{array}{l}\text {... el gobierno no acaba de subir los pasajes del transporte público } \\
\text { y de carga en todo el país esa es la verdadera medida que tomó el } \\
\text { gobierno hace pocos días en materia económica ... }\end{array}$ \\
\hline
\end{tabular}
Contexto: Entrevista a la alcaldesa -RTS ${ }^{5}$

Cuadro 1. Deixis

\subsection{Referencias e Inferencias Espacial}

\subsubsection{Referencias} ... es un placer estar con ustedes ahora en esta ...

\footnotetext{
${ }^{1}$ https://www.youtube.com/watch?v=RXUL05XIj6s

2 https://www.youtube.com/watch?v=RXUL05XIj6s

${ }^{3}$ https://www.youtube.com/watch?v=3DUNxbzi-oU

${ }^{4}$ https://www.youtube.com/watch?v=vPJQKA5rJMg

${ }^{5}$ https://www.youtube.com/watch?v=RXUL05XIj6s
} 


\begin{tabular}{ll}
\hline & Análisis: Hace referencia a los periodistas de Radio Élite. \\
\hline & Contexto: Cynthia Viteri - enlace radio centro \\
\hline Ejemplo 08 & $\ldots$ esta nueva modalidad en exteriores que ... \\
\hline & Análisis: Se refiere a los exteriores de la alcaldía de Guayaquil. \\
\hline & Contexto: Dra. Cynthia Viteri - Radio ÉliteiError! Marcador no definido. \\
\hline
\end{tabular}

\subsubsection{Inferencias}

\begin{tabular}{ll}
\hline Ejemplo 09 & ... qué bien de aquí mire un tema que realmente me resalta el \\
presupuesto del 40 por ciento del presupuesto será destinado \\
para la obra social esto se decidió en la última sesión esto va a ser \\
replicado todos los años permanente me he puesto del municipio \\
& 40 por ciento para la obra social permanentemente ... \\
& Análisis: El porcentaje destinado es el suficiente para esta \\
& actividad. \\
& Análisis: El presupuesto ya ha sido aprobado. \\
& Análisis: Un estudio estadístico ha sido hecho previo a la \\
& aprobación del presupuesto. \\
& Contexto: Cynthia Viteri - enlace radio centro ${ }^{\text {iError! Marcador no definido. }}$ \\
\hline
\end{tabular}

\subsubsection{Nombre y referentes}

\begin{tabular}{ll}
\hline Ejemplo 10 & $\ldots$ la doctora Cynthia Viteri, un gusto ... \\
\hline & Análisis: En referencia a la Alcaldesa de Guayaquil \\
\hline Contexto: Dra. Cynthia Viteri - Radio Élite ${ }^{\text {iEror! Marcador no definido. }}$ \\
\hline Ejemplo 11 & ... haciendo matriz desde Radio Élite nos ... \\
\hline & Análisis: Radio Élite entrevista a Cynthia Viteri \\
\hline & Contexto: Dra. Cynthia Viteri - Radio Élite ${ }^{\text {EError! Marcador no definido. }}$ \\
\hline
\end{tabular}

\subsubsection{Co-texto}

Ejemplo $12 \quad$... está más en la mira de convenciones de eventos internacionales que dejan, desde el taxista desde el transporte urbano económico, desde la gastronomía hoteles y todo dinero en el bolsillo de los guayaquileños de la gente que trabaja aquí, es un evento importantísimo que lo va a tener Guayaquil ...

Análisis: En el evento se refiere a los ciudadanos de la ciudad de Guayaquil

Contexto: Dra. Cynthia Viteri - Radio ÉliteiError! Marcador no definido.

\subsubsection{Referencia anafórica}

\begin{tabular}{ll}
\hline Ejemplo 13 & ... su sueño y coincidió con su cumpleaños ... \\
\hline & Análisis: Hace referencia a Sergio \\
\hline Ejemplo 14 & $\begin{array}{l}\text { Contexto: Cynthia Viteri - Radio Élite es querr! Marcador no definido. } \\
\text { por su cumpleaños ... }\end{array}$ \\
\hline
\end{tabular}

${ }^{6}$ https://www.youtube.com/watch?v=vPJQKA5rJMg 
Análisis: Hace referencia a Sergio

Contexto: Dra. Cynthia Viteri - Radio Éliteierror! Marcador no definido.

Cuadro 2. Referencias e Inferencias

\subsection{Presuposiciones y declaraciones}

\subsubsection{Potencial}

Ejemplo 15

... este yo quiero que sea un programa permanente, poder ir realizando sueños a niños que tengan algún tipo de enfermedad catastrófica y que puedan realizar su sueño ...

Contexto: Dra. Cynthia Viteri - Radio ÉliteiError! Marcador no definido.

\subsubsection{Existencial}

Ejemplo 16

... pero que le he propuesto al gobierno central es su responsabilidad el tema de las drogas ...... su sueño y coincidió con su cumpleaños ...

Contexto: Dra. Cynthia Viteri - Radio ÉliteiError! Marcador no definido.

Ejemplo 17

... y un mensaje final a las mamás que tenemos o que tienen yo

tengo nietos pequeños a las mamás que tienen sus hijos

pequeños nosotros tenemos que llegar antes ...

Contexto: Dra. Cynthia Viteri - Radio ÉliteiError! Marcador no definido.

\subsubsection{Factive}

Ejemplo 18

... también hay otra posibilidad vamos a tomar la que mayor gente cubra mayor necesidad allá también hay una que sale ...

Contexto: Dra. Cynthia Viteri - Radio ÉliteiError! Marcador no definido.

\subsubsection{Lexical}

Ejemplo 19

... ciertamente ya estamos, como dice la canción no hay cama para tanta gente, y es cada vez que estamos un poquito más apretados

Contexto: Dra. Cynthia Viteri - Radio ÉliteiError! Marcador no definido.

\subsubsection{Estructural}

Ejemplo 20 ... lo que sí, personalmente sigo insistiendo, es la renovación de la metrovía siguen muchos ...

Contexto: Dra. Cynthia Viteri - Radio ÉliteiError! Marcador no definido.

\subsubsection{No-Factive}

Ejemplo $21 \quad$... este yo quiero que sea un programa permanente, poder ir realizando sueños a niños que tengan algún tipo de enfermedad catastrófica y que puedan realizar su sueño ...

Contexto: Dra. Cynthia Viteri - Radio ÉliteiError! Marcador no definido.

\subsubsection{Factual}

Ejemplo 22

... la gente está pagando 35 centavos por rodar en estos buses que son eléctricos y que tienen aire acondicionado por ir más cómodos sin ningún problema ... 


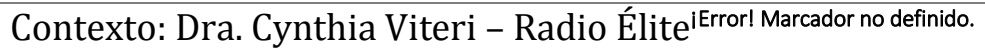

Cuadro 3. Presuposiciones y Declaraciones

\subsection{Cooperación}

4.4.1 Máximas de cantidad

Ejemplo $23 \quad$ Gabriela Uquillas: ¿Cuáles son las alternativas que ha propuesto el municipio y qué tienen en mente en relación con la vialidad la vialidad del transporte pesado?

Cynthia Viteri: "esa vía no aguanta más tránsito la vía la costa no aguanta más tránsito y recuerda que también por la misma vía está proyectado el aeropuerto cuyos estudios ya estoy retomando y la nueva terminal satélite terrestre también vía la costa es un polo de desarrollo y el único sitio para dónde va a crecer ..."

Análisis: Cynthia al responder la pregunta por parte de la reportera da información con muchos detalles.

Contexto: Cynthia Viteri conversó con 5 ciudadanos sobre el futuro de Guayaquil ${ }^{7}$

Ejemplo 24

Luisa - Entrevistadora RTS: ¿Cuál va a ser específicamente el aporte que nosotros sumaremos como ciudad con usted como la primera autoridad de Guayaquil a la cabeza para contribuir a la seguridad del puerto principal?

Cynthia Viteri: "apoyar a la policía nacional tanto con recursos con tecnología y con infraestructura si podemos resumir cuál es la propuesta y la acción desde el municipio de Guayaquil que como tú sabes luisa no le compete la seguridad competencia exclusiva del estado hemos llegado a un convenio con el ministerio del interior- y también con el consejo de la judicatura y la fiscalía general del estado..."

Análisis: Cynthia al responder la pregunta da muchos detalles sobre qué instituciones toman parte de la seguridad y también sobre los convenios realizados para solventar el problema de la inseguridad.

Contexto: Entrevista alcaldesa -RTS ${ }^{8}$

\subsubsection{Máximas de relación}

\section{Ejemplo 25}

Gabriela Uquillas: $¿$ Cuáles son las alternativas que ha propuesto el municipio y qué tienen en mente en relación con la vialidad la vialidad del transporte pesado?

Cynthia Viteri: "esa vía no aguanta más tránsito la vía la costa no aguanta más tránsito y recuerda que también por la misma vía está proyectado el aeropuerto cuyos estudios ya estoy retomando y la nueva terminal satélite terrestre también vía la costa es un polo de desarrollo y el único sitio para dónde va a crecer ..."

Análisis: Cynthia al responder la pregunta por parte de la reportera da información con muchos detalles.

\footnotetext{
${ }^{7}$ https://www.youtube.com/watch?v=SuGVMJ0JI6k

8 https://www.youtube.com/watch?v=RXUL05XIj6s
} 


\begin{tabular}{ll}
\hline & $\begin{array}{l}\text { Contexto: Cynthia Viteri conversó con } 5 \text { ciudadanos sobre el } \\
\text { futuro de Guayaquil }\end{array}$ \\
\hline Ejemplo 26 & Entrevistadora RTS: ¿Cuál va a ser específicamente el aporte que \\
& nosotros sumaremos como ciudad con usted como la primera \\
& autoridad de Guayaquil a la cabeza para contribuir a la seguridad \\
del puerto principal? & \\
& Cynthia Viteri: “apoyar a la Policía Nacional tanto con recursos \\
& con tecnología y con infraestructura si podemos resumir cuáles \\
& propuestas y la acción desde el Municipio de Guayaquil que como \\
& tú sabes Luisa no le compete la seguridad competencia exclusiva \\
& del Estado hemos llegado a un convenio con el Ministerio del \\
& Interior- y también con el Consejo de la Judicatura y la Fiscalía \\
& General del Estado...” \\
\hline Análisis: Cynthia Viteri responde la pregunta, pero de una manera \\
indirecta al brindar mucha información. \\
Contexto: Entrevista alcaldesa -RTS ${ }^{10}$
\end{tabular}

Cuadro 4. Cooperación

\subsection{Implicaturas}

4.5.1 Escalar

Ejemplo $27 \quad \ldots$ un gusto un, placer estar con ustedes ahora en esta nueva modalidad en exteriores ...

Contexto: Dra. Cynthia Viteri - Radio Élite ${ }^{11}$

Ejemplo $28 \quad \ldots$ haciendo matriz desde radio Élite nos represa en varias emisoras un abrazo a todos pues sí a la orden yo quería tomar un tema de la semana ...

Contexto: Dra. Cynthia Viteri - Radio Élite ${ }^{12}$

\subsubsection{Conversacional}

Ejemplo $29 \quad$... pero su sueño era ser alcalde por un día y hemos establecido un programa en la dirección de acción social, Boris para hacer realidad los sueños de los niños sobre todo los niños que padecen enfermedades muy duras como cáncer hay otros niños también que tienen otro tipo de enfermedades catastróficas muy muy duras y queremos hacer este programa se llama fin consiste en que si en este caso Sergio había tenido el sueño es ser alcalde pueda estar presente en una sesión dio inicio a la sesión ... Contexto: Dra. Cynthia Viteri - Radio Élite ${ }^{\mid \mathrm{E} \text { Error! Marcador no definido. }}$

\subsubsection{Convencional}

\footnotetext{
${ }^{9}$ https://www.youtube.com/watch?v=SuGVMJ0JI6k

${ }^{10} \mathrm{https}$ // $\mathrm{www}$.youtube.com/watch?v=RXUL05XIj6s

11 https://www.youtube.com/watch?v=LrpIEnRs7NY

12 https://www.youtube.com/watch?v=LrpIEnRs7NY
} 


\begin{tabular}{ll}
\hline Ejemplo 30 & $\begin{array}{l}\text {... presenció todo lo trataban como alcalde desde el principio, } \\
\text { desde que llegó al municipio los policías municipales que se le } \\
\text { cuadraban cuando él pasaba ... }\end{array}$ \\
\hline Contexto: Dra. Cynthia Viteri - Radio Élite & RError! Marcador no definido. \\
\hline
\end{tabular}

Cuadro 5. Implicaturas

\subsection{Actos de discurso}

4.6.1 Actos locucionarios

Ejemplo $31 \quad$... gracias a esas mil cien cámaras, nosotros podemos ver dónde hay accidentes de tránsito incendio y situaciones de riesgo y también vemos asaltos vemos agresiones vemos bandas y qué hemos hecho nosotros, hasta ahora la policía si tienen algún representante voluntariamente porque va siempre van hay que decirlo claro ellos van en la corporación

Análisis: El propósito de este discurso es "informar"

Contexto: Dra. Cynthia Viteri - Enlace Radio Águila ${ }^{13}$

Ejemplo32 ... otra buena noticia John y para todos los que me escuchan es que hicimos juntos con el Banco del Pacífico con su con quien lo dirige Efraín Vieira el convenio del banco de oportunidades de manera distinta que hicimos ahora los créditos no van no son hasta 500 dólares van de 500 dólares hasta 1500 dólares eso lo logramos en estos 60 días ...

Análisis: El propósito de este contexto es "informar"

Contexto: Dra. Cynthia Viteri - Enlace Radio Águila ${ }^{\text {EError! Marcador no }}$ definido.

\subsubsection{Actos ilocucionarios}

Ejemplo $33 \quad$... gracias a esas mil cien cámaras nosotros podemos ver dónde hay accidentes de tránsito incendio y situaciones de riesgo y también vemos asaltos vemos agresiones vemos bandas y que hemos hecho nosotros hasta ahora la policía si tienen algún representante voluntariamente porque va siempre van hay que decirlo claro ellos van en la corporación ...

Análisis: El propósito de este discurso es dar a conocer a la ciudadanía que el asunto de la seguridad es una de las prioridades para la alcaldesa.

Contexto: Dra. Cynthia Viteri - Enlace Radio Águila ${ }^{\text {iError! Marcador no }}$ definido.

Ejemplo $34 \quad$... otra buena noticia John y para todos los que me escuchan es que hicimos juntos con el Banco del Pacífico con su con quien lo dirige Efraín Vieira el convenio del banco de oportunidades de manera distinta que hicimos ahora los créditos no van no son hasta 500 dólares van de 500 dólares hasta 1500 dólares eso lo logramos en estos 60 días ...

13 https://www.youtube.com/watch?v=A8gZRwVIDuU 
Análisis: El propósito de este contexto es comunicar a los guayaquileños sobre las nuevas posibilidades de créditos, a las cuales ellos pueden acceder en el banco del Pacífico.

Contexto: Dra. Cynthia Viteri - Enlace Radio Águila ${ }^{\text {EError! Marcador no }}$ definido.

\subsubsection{Actos perlocucionarios}

\begin{tabular}{|c|c|}
\hline \multirow[t]{3}{*}{ Ejemplo 35} & $\begin{array}{l}\text {... gracias a esas mil cien cámaras nosotros podemos ver dónde } \\
\text { hay accidentes de tránsito incendio y situaciones de riesgo y } \\
\text { también vemos asaltos vemos agresiones vemos bandas y que } \\
\text { hemos hecho nosotros hasta ahora la policía si tienen algún } \\
\text { representante voluntariamente porque va siempre van hay que } \\
\text { decirlo claro ellos van en la corporación ... }\end{array}$ \\
\hline & $\begin{array}{l}\text { Análisis: El efecto de este discurso es tranquilizar a los ciudadanos } \\
\text { sobre el asunto de la delincuencia, diciéndoles que se han } \\
\text { incorporado más opciones de seguridad para la ciudadanía. }\end{array}$ \\
\hline & $\begin{array}{l}\text { Contexto: Dra. Cynthia Viteri - Enlace Radio Águila } \\
\text { definido. }\end{array}$ \\
\hline \multirow[t]{3}{*}{ Ejemplo 36} & $\begin{array}{l}\text {... otra buena noticia John y para todos los que me escuchan es que } \\
\text { hicimos juntos con el Banco del Pacífico con su con quien lo dirige } \\
\text { Efraín Vieira el convenio del banco de oportunidades de manera } \\
\text { distinta que hicimos ahora los créditos no van no son hasta } 500 \\
\text { dólares van de } 500 \text { dólares hasta } 1500 \text { dólares eso lo logramos en } \\
\text { estos } 60 \text { días ... }\end{array}$ \\
\hline & $\begin{array}{l}\text { Análisis: El efecto que causa esta información es incentivar a los } \\
\text { guayaquileños aplicar a las nuevas oportunidades de créditos } \\
\text { bancarios }\end{array}$ \\
\hline & $\begin{array}{l}\text { Contexto: Dra. Cynthia Viteri - Enlace Radio Águila } \\
\text { definido. }\end{array}$ \\
\hline
\end{tabular}

Cuadro 6. Actos de discurso

\subsection{Clasificación del discurso}

4.7.1 Representativas

Ejemplo $37 \quad$... yo creo que el embarazo y lo digo solo de manera particular en mi caso específico me salvó de otro destino me salvó y otro destino porque a esa edad a mí no me importaba nada ...

Análisis: Cynthia Viteri, desde su punto de vista, indica que de una u otra manera el embarazo marcó su vida de manera muy positiva para su vida profesional. Contexto: CatolicaTV - Perfil Cynthia Viteri ${ }^{14}$

Ejemplo $38 \quad \ldots$ yo creo que por eso Dios nos guía y podemos o tratamos como nos esforzamos por hacer las cosas bien cuesta mucho trabajo ... hasta 500 dólares van de 500 dólares hasta 1500 dólares eso lo logramos en estos 60 días ...

${ }^{14}$ https://www.youtube.com/watch?v=FpGT43wQOXI 
Análisis: En esta parte del video Viteri comparte su fe en Dios, quien para ella es un pilar muy importante en su vida entera.

Contexto: Cynthia Viteri, la alcaldesa: "Guayaquil es mi corazón" MarielaTV ${ }^{15}$

\subsubsection{Expresivo}

Ejemplo 39
... el trabajo más emocionante lo que más me gusta de todo esto es la calle me encanta poder regresar a la calle me asfixia en los escritorios ...

Análisis: Cynthia Viteri expresa que ama estar en las calles, en lugar de estar en una oficina.

Contexto: Cynthia Viteri, la alcaldesa: "Guayaquil es mi corazón" MarielaTV $^{15}$

Ejemplo $40 \quad$... no me gustaba la locución fui muy poco tiempo locutora porque era muy tenso tener que estar arreglada, pintada, maquillada vestida a una hora determinada ...

Análisis: La Alcaldesa de Guayaquil comparte que ella trabajó como locutora por un tiempo, pero fue una actividad que no le gustaba.

Contexto: Cynthia Viteri, la alcaldesa: "Guayaquil es mi corazón" MarielaTV ${ }^{15}$

\subsubsection{Directivos}

Ejemplo 41

... la democracia la democracia no caerá en las calles de Guayaquil y menos un 9 de octubre que viva Guayaquil ...

Análisis: Con este discurso Cynthia Viteri intenta persuadir a los guayaquileños para no permitir que personas ajenas a la ciudad lleguen a destruir lo que ellos han construido por años.

Contexto: Esto dijo Cynthia Viteri en su intervención en la marcha por la paz de Guayaquil - Teleamazonas ${ }^{16}$

Ejemplo $42 \quad$... somos la policía que aguantó humillación y golpe para no caer en la provocación somos los agentes de la gn, de los metropolitanos, de justicia y vigilancia del mercado del municipio de Guayaquil que terminaron ...

Análisis: En este video Cynthia Viteri mediante el discurso en la marcha por la paz, motiva a sus ciudadanos a cuidar de su ciudad y no permitir dejarse caer en la provocación que puedan causar daños irreparables en Guayaquil.

Contexto: Esto dijo Cynthia Viteri en su intervención en la marcha por la paz de Guayaquil - Teleamazonas ${ }^{16}$

\subsubsection{Comisivas}

Ejemplo 43

... llegamos a un acuerdo que beneficia a los guayaquileños porque eso es lo que me compete por un lado nosotros haremos la desintoxicación con medicamentos aprobados por el ministerio

15 https://www.youtube.com/watch?v=sUW2MDNTD7c

16 https://www.youtube.com/watch?v=lKe9TkSfM7E 


\begin{tabular}{ll}
\hline psiquiátrico y en personal psicólogos y psiquiatras también \\
nuestra primera clínica de rehabilitación móvil ... \\
Análisis: La Dra. Viteri indica que tiene un plan a futuro como es \\
lograr la desintoxicación de jóvenes drogadictos, el cual se \\
cumplirá en un futuro no muy lejano. \\
\hline Contexto: Dra. Cynthia Viteri - Enlace radial Radio Centro ${ }^{17}$ \\
\hline Ejemplo 44 ese es nuestro nuevo plan y llegaré a un acuerdo porque sé \\
que lo haremos con el ministerio de educación para poder ir a las \\
escuelas fiscales y prevenir en nuestros niños que sean los \\
próximos que caigan en este desastre ... \\
\hline Análisis: En esta entrevista la alcaldesa Cynthia da a conocer a su \\
audiencia el plan que tiene para mejorar la calidad de Educación. \\
\hline Contexto: Entrevista alcaldesa Cynthia Viteri - RTS ${ }^{18}$
\end{tabular}

Cuadro 7. Clasificación del discurso

\subsection{Amabilidad e interacción}

4.8.1 Amabilidad

Ejemplo $45 \quad$... llegar nosotras y nosotros antes que ellos al corazón a la mente y a la inteligencia en nuestro sitio en un abrazo para ustedes ... Análisis: Ustedes, se refiere a la audiencia de Radio Élite Contexto: Dra. Cynthia Viteri - Radio Élite ${ }^{19}$

Cuadro 8. Amabilidad e interacción

Después del análisis con cada uno de los ejemplos anteriormente expuestos y con la observación de los videos de la Dra. Cynthia Viteri, se observa algunas de las características femeninas lingüísticas propias de ella, las mismas que se evidencian en los siguientes ejemplos tomados de sus videos.

\subsection{Prosodia y elementos paralingüísticos}

Ejemplo $01 \quad$... de esta alianza en donde el Municipio de Guayaquil iba a colaborar para ser puntual (finales ascendentes) eficientemente en la comunicación inmediata (cambio de entonación más enfática) de un hecho delincuencial para que la policía actúe de manera efectiva y reduzca sus tiempos para llegar (finales ascendentes) inmediatamente al sitio del hecho (cambio de entonación más enfática) nuestra colaboración era tecnológica y vamos a tener un solo sistema (finales ascendentes) el 911 se iba a integrar al nuestro y de esa manera cuando alguien era víctima y un hecho delictivo el patrullero más cercano con gps que también les íbamos a dar (cambio de tonos de voz con tendencias a tonos más agudos) nosotros a los patrulleros o nuestro personal iba a ir inmediatamente en auxilio el ciudadano ese era nuestro claro qué pasó después de las conversaciones siguieron (cambio de tonos de voz con tendencias a tonos más

17 https://www.youtube.com/watch?v=vPJQKA5rJMg

18 https://www.youtube.com/watch?v=RXUL05XIj6s

${ }^{19} \mathrm{https}: / /$ www.youtube.com/watch?v=LrpIEnRs7NY 


\begin{tabular}{ll}
\hline & agudos) dándose semanas en donde se reunieron delegados de \\
& cada una de estas funciones y ... \\
Contexto: ¡Con Guayaquil no se juega! ${ }^{20}$ \\
\hline Ejemplo 02 \\
(alargamientos vocálicos), un mensaje escrito de la ministra del \\
interior nos decía como guayaquileños, que estaba lista para \\
firmar para que Guayaquil, el municipio (alargamientos \\
vocálicos), que ella sabe porque es abogada, para que el \\
municipio, se haga cargo de la seguridad integral (cambios de \\
entonación más enfáticos y alargamientos vocálicos) de la \\
ciudad ... \\
Contexto: ¡Con Guayaquil no se juega!
\end{tabular}

4.9.1 Morfosintaxis

Ejemplo $03 \quad$... de firmar el convenio señores luego de que ya todos se habían puesto de acuerdo en una mesa con representantes del ministerio del interior- aceptando este engranaje de fuerzas y nosotros poniendo toda nuestra colaboración (haciendo referencia a los miembros del Consejo Municipal de Guayaquil) al día anterior de la firma es decir al día anterior del 25 de julio el ministerio del interior- mandó un nuevo convenio desconociendo todos los acordados por todas las autoridades en esa mesa jugando con Guayaquil ... Contexto: ¡Con Guayaquil no se juega! ${ }^{20}$

Ejemplo $04 \quad$... nos mandaron a decir (haciendo referencia a los miembros del Consejo Municipal de Guayaquil) que estaban de acuerdo en el convenio inicial, dio sorpresa de mala fe con absoluta mala fe y queriendo que Guayaquil caiga en una trampa, un mensaje escrito de la ministra del interior nos decía como guayaquileños (haciendo referencia a los miembros del Consejo Municipal de Guayaquil), que estaba lista para firmar para que Guayaquil, el municipio, que ella sabe porque es abogada, para que el municipio se haga cargo de la seguridad integral de la ciudad ...

Contexto: ¡Con Guayaquil no se juega! ${ }^{20}$

\subsubsection{Léxico}

\begin{tabular}{ll}
\hline Ejemplo 05 & ... así es que no hay tiempo, la vida tan pequeñita cambia Mariela \\
tan pequeñita hace poco éramos veinteañeras ahora yo soy más \\
de 50,53 tú no ... \\
Análisis: En este contexto se usa un diminutivo para hacer \\
referencia al gran afecto y emoción que le da recordar el tiempo \\
que era joven. \\
Contexto: Cynthia Viteri, la alcaldesa: "Guayaquil es mi corazón" - \\
MarielaTV
\end{tabular}

${ }^{20} \mathrm{https} / / / \mathrm{www}$. youtube.com/watch?v=nCKEesewxfk

${ }^{21} \mathrm{https}: / /$ www.youtube.com/watch?v=sUW2MDNTD7c 


\begin{tabular}{ll}
\hline Ejemplo 06 & ... me incorporé y cuando ya me incorporé ya tenía tres hijos \\
& Johana Julie y Juliana y ahora a ellos ya tienen la 36 el otro 30 la \\
otra 28 y mis bebés que son mis gemelos de 18 años varón y mujer \\
que ya se gradúa en este año ingresar a la universidad y que ahora \\
son desde que nacieron son como mis niños pequeñitos y no \\
termino de verlos crecer nunca creo ... \\
Análisis: En este video se habla específicamente de su familia, su \\
vida por tanto, se puede observar el afecto y la emoción al \\
momento de expresarse de su familia, así como también utiliza \\
diminutivos para enfatizar el gran amor por sus hijos. \\
\hline Contexto: CatólicaTV - Perfil Cynthia Viteri ${ }^{22}$ \\
\hline
\end{tabular}

\subsubsection{Organización temática textual}

Ejemplo 07 ... lo único que no puedes hacer es quedarte sentado esperando a ver qué haces no para esparcir estar estudiando ya el pre de una carrera y sé pero que sigue en el camino vas a saber pero y en el análisis que pasa en la retrospectiva que pasa en pensar y hacer una yo no soy de eso, yo tengo como tu, camino, camino, camino, camino, si hay una y decidido por qué camino ir exacto, exacto pero todo rápido todo el vuelo así es que no hay tiempo la vida tan pequeñita cambia bailar tan pequeñita hace poco éramos ingeniera ahora yo soy más de 50 ...

Análisis: En esta parte se puede ver con claridad las interrupciones por parte de las dos personas (Cynthia y Mariela), características lingüísticas femeninas, las mismas que indican cooperación e indica comprensión del tema tratado.

Contexto: Cynthia Viteri, la alcaldesa: "Guayaquil es mi corazón" MarielaTV ${ }^{23}$

Ejemplo $08 \quad$... respondiendo a tu pregunta ningún acto de violencia venga de donde venga y si se da pues va a ser sancionado no solamente administrativamente sino que si el caso lo amerita vaso tras día hasta entonces en general los agentes de la policía metropolitana o sea lo que se analiza van a ir destinados a término seguridad en general es un programa piloto y vamos a seguir cuidando el orden de la ciudad y protegiendo a la gente de la delincuencia pero de manera cómo lo deben hacer cada funcionario del Municipio ...

Análisis: En este video se puede ver claramente las interrupciones en las preguntas de entrevista, sin perder el hilo del tema, lo cual indica cooperación y comprensión de dichas preguntas.

Contexto: Las propuestas de Cynthia Viteri para la Alcaldía de Guayaquil - Teleamazonas ${ }^{24}$

${ }^{22}$ https://www.youtube.com/watch?v=FpGT43wQOXI

${ }^{23} \mathrm{https}: / /$ www.youtube.com/watch?v=sUW2MDNTD7c

${ }^{24} \mathrm{https}$ ://www.youtube.com/watch?v=Z7e4mA8XUdg 


\subsubsection{Elementos no verbales (cinesia y proxemia)}

\begin{tabular}{ll}
\hline Ejemplo 09 & $\begin{array}{l}\text { Análisis: En este video se puede evidenciar la postura de piernas } \\
\text { cruzadas y la utilización de las manos para expresarse y responder } \\
\text { las preguntas. }\end{array}$ \\
\hline Contexto: Entrevista alcaldesa Cynthia Viteri - RTS & \\
\hline Ejemplo 10 & $\begin{array}{l}\text { Análisis: Es muy claro la proximidad entre Mariela y Cynthia al } \\
\text { momento de hablar, así como también las piernas cruzadas } \\
\text { durante toda la entrevista, movimiento de manos y gestos faciales. }\end{array}$ \\
\hline & $\begin{array}{l}\text { Contexto: Cynthia Viteri, la alcaldesa: "Guayaquil es mi corazón" - } \\
\text { MarielaTV }\end{array}$ \\
\hline
\end{tabular}

Cuadro 9. Prosodia y elementos paralingüísticos

\subsection{Características del análisis}

Existen otros aspectos del significado que dependen más del contexto y las intenciones comunicativas de los hablantes, pues debe considerarse que "la comunicación depende no solo de reconocer los significados de las palabras, sino también de lo que los hablantes quieren decir en ese momento concreto" (Álvarez, 2019, pág. 15). La disciplina que nos ayuda a entender estos significados es la Pragmática, es decir, la interpretación de los significados en contexto de los elementos extralingüísticos y de las situaciones comunicativas.

Se debe tener en cuenta el significado invisible, "reconocer lo que se quiere decir incluso cuando no se dice" (Yule, 2006, pág. 112). Se estudian los dos tipos de contexto principales: el lingüístico (contexto) y el físico. Bastante relacionado con este último está el concepto de deixis, como se puede observar en el Cuadro 1; a propósito del concepto de referencia como en el Cuadro 2, vemos que no son las palabras las que refieren por sí mismas, sino las personas. No hay que confundirla con la inferencia, que se refiere al proceso por el cual se derivan conclusiones a partir de proposiciones.

La anáfora, que consiste en la repetición de una o varias palabras al principio de dos o más líneas de los versos de una estrofa o de un enunciado, corresponde a la segunda vez que se utiliza una referencia, la primera es el antecedente; mientras que las presuposiciones son lo que un hablante supone que es verdad o conocido por un oyente, como por ejemplo en el Cuadro 3. Se prosigue con los actos de habla y se estudia cómo su uso tanto directo como indirecto se relaciona con la cortesía o imagen, ya sea negativa o positiva. El análisis del discurso se ocupa del estudio del lenguaje en los textos y en las conversaciones. En el Cuadro 4 se observa el análisis de la conversación y cómo pueden actuar los participantes en la toma de turnos, manejo de puntos finales, etc. Se analiza también el principio de cooperación con las cuatro máximas de Grice:

a) Máxima de cantidad, cuando se proporciona información necesaria para el propósito de la conversación.

b) Máxima de calidad, la información proporcionada tiene que ser veraz.

c) Máxima de relación, si la contribución de información es pertinente al tema de la conversación.

d) Máxima de modo, la información debe ser clara y breve para evitar ambigüedades.

25 https://www.youtube.com/watch?v=RXUL05XIj6s

26 https://www.youtube.com/watch?v=sUW2MDNTD7c 
A diferencia de las reglas sintácticas y semánticas, los principios pragmáticos y las convenciones funcionan tanto cuando aparentemente son violados, "cuando el orador S cuenta con el oyente $\mathrm{H}$ para reconocer la violación aparente y para realizar el ajuste contextual apropiado, como cuando se observan o se violan con ostentación" (Horn \& Ward, 2006, pág. 8). Por ello, ni el principio de cooperación ni las máximas correspondientes están diseñadas como prescripciones para acciones éticas o como observaciones etnográficas, una aproximación más precisa es verlas como configuraciones predeterminadas cuya conciencia mutua es compartida por el habla de los participantes.

\subsection{Características del lenguaje femenino}

A través de la observación y la entrevista es posible recopilar información sobre cómo se realizan los actos de habla en una comunidad de habla determinada, como por ejemplo, en el lugar de trabajo en donde se realizan solicitudes a colegas, rechazan solicitudes de personas de mayor estatus y agradecen a las personas en servicio, como los trabajadores de la cafetería o custodios, ejemplo en el Cuadro 5; la manera de señalarlo tiene que estar relacionado con "lo que dicen/cómo lo dicen, velocidad de entrega y tono de voz/ y su comportamiento no verbal tal como lo dicen las expresiones faciales, postura corporal y gestos" (Ishihara \& Cohen, 2010, pág. 230). Según el idioma y la cultura, puede ser estratégico para el orador ajustar la ejecución del acto de habla según la edad, el estado relativo o el género del oyente, esto se puede observar en el Cuadro 6. El orador también necesitaría saber qué significa pedir algo, como por ejemplo un automóvil, en este contexto particular, es decir, cuán grande es la imposición que se considera en esa cultura, si un automóvil es una necesidad diaria o un lujo y en ese contexto específico, pedir prestado un automóvil nuevo y relativamente valioso en comparación con un automóvil antiguo "de repuesto".

Por lo tanto, "el discurso natural recuperado a través de los datos del corpus revela que podría tomar varios turnos para que la interacción se resuelva por sí misma, de una forma u otra" (Ishihara y Cohen, 2010, pág. 238). La realización de actos efectivos de habla se relaciona no solo en base al conocimiento de lo que es apropiado o no en determinadas culturas, sino también del conocimiento de la lengua para hacerlos de manera apropiada, como ejemplo se tiene el Cuadro 7.

Como algunas de las principales características del lenguaje femenino, se mencionan las siguientes:

Deseo de incluir como iguales en el discurso a las otras personas participantes, haciendo énfasis en la relación con ellas; atención extrema a las palabras ajenas y a los mensajes externos - verbales o no verbales; la indicación expresa de su escucha e implicación en la conversación; una búsqueda de intimidad; el desarrollo de los temas de forma cooperativa. Mientras que para el estilo masculino menciona: el deseo de afirmarse frente a las demás personas participantes; su participación se mide más por el tipo de intervención que por la demostración de atención; una búsqueda de objetividad y distanciamiento; el desarrollo de los temas de forma individualista y frecuentemente competitiva; una expresión aparentemente hostil de la solidaridad masculina (Bengoechea, 1996, pág. 3).

El lenguaje corporal de las mujeres no es tan diferente del lenguaje corporal de los hombres. Todos usan las mismas expresiones faciales $y$, en su mayor parte, el mismo lenguaje 
corporal para expresar cómo se sienten, cómo atraen el uno al otro, cómo buscan atención, y cuáles son sus signos de ansiedad.

Se estima que la "mujer puede pronunciar sin esfuerzo una media de entre 6.000 y 8.000 palabras diarias" (Pease \& Pease, 2001, pág. 98). Además de que puede utilizar adicionalmente entre 2.000 y 3.000 sonidos para comunicarse, y unos 10.000 gestos y expresiones faciales. Un total de más de 20.000 unidades de comunicación para transmitir un determinado mensaje. Mientras que el hombre, en cambio, emplea sólo entre $2.000 \mathrm{y}$ 4.000 palabras, entre 1.000 y 2.000 sonidos y emite como mucho 3.000 señales de lenguaje corporal. Es decir que su media diaria asciende a cerca de 7.000 unidades de comunicación. Un tercio de lo que emiten las mujeres.

Además, se añade que, en el momento de comunicar, "las mujeres piensan en voz alta y consideran este tipo de comportamiento como un gesto de amistad, pues hablando permiten que los demás compartan sus pensamientos" (Pease \& Pease, 2001, pág. 99). Más aún, liberan sus sentimientos para así poder afrontarlos mejor. Sin embargo, los hombres no suelen comprender esta actitud y lo entienden como que la mujer está pasándoles una lista de problemas para solucionar lo antes posible. Pero ellas no esperan respuestas, sino disponer de alguien que las escuche con atención o que confirme lo que ellas dicen, como ejemplo se tiene el Cuadro 8.

Se puede señalar que, para los hombres, "la conversación es la manera de negociar su estatus en el grupo y evitar que la gente empuje a su alrededor. El varón utiliza la charla para preservar su independencia" (Tannen, 1991, pág. 21). Las mujeres, en cambio, usan la conversación para negociar la cercanía y la intimidad; de hecho, hablar es la esencia de la intimidad, por lo que ser mejores amigos significa sentarse y hablar. Para los chicos, en cambio, las actividades, el hacer cosas juntos, son centrales. Simplemente sentarse y hablar no es una parte esencial de la amistad. Además, para las mujeres, hablar de problemas constituye la esencia de la conexión, ver Cuadro 9. Los hombres, sin embargo, cuando escuchan los problemas, lo interpretan como una solicitud de asesoramiento, por lo que responden con una solución.

Si se busca una explicación a lo anterior, se podría mencionar de manera antropológica que "los hombres evolucionaron como cazadores de alimentos, no como comunicadores" (Pease \& Pease, 2001, pág. 89). En cambio, las mujeres solían pasar sus días en compañía de otras mujeres y niños del grupo y por ello desarrollaron la habilidad de comunicarse con éxito para poder mantener relaciones.

La evolución desde las actividades de subsistencia fundamentalmente individualistas de los primates superiores a la compleja empresa cooperativa de la caza y la recolección requería seguramente una comunicación eficiente. Una hipótesis popular del desarrollo del lenguaje incluye la idea de que en una primera etapa habría sido un lenguaje de gestos --gesticular, recuérdese, es algo que los seres humanos hacen con frecuencia, especialmente cuando no encuentran las palabras adecuadas.

\section{Conclusiones}

El lenguaje femenino se manifiesta en la Dra. Cynthia Viteri de una manera muy clara y evidente, como son el uso de la prosodia, elementos paralingüísticos, lenguaje corporal, una entonación más enfática, alargamientos vocálicos, también realiza cambio de tono de voz cuando necesita enfatizar algo muy importante. Así como también se observó un uso frecuente de vocalizaciones como ( $\mathrm{mmm}$, ajá o similares) para indicar que está siguiendo el diálogo o alguna entrevista. 
Otro elemento importante sobre el lenguaje femenino es la morfosintaxis en donde hace uso de la segunda persona del singular y de la primera persona del plural, con la finalidad de incluir a la persona o a las personas con quienes ella está interactuando. Además, se observa el uso frecuente de oraciones interrogativas y exclamativas (¿nooo?, ¿verdaaad?, ¿eeeh?, ¿a que siií?, ¿no te pareeece?, etc.).

En los diálogos presentados por la Dra. Viteri el vocabulario es referente a los ámbitos privados como son la familia y el hogar; mientras que los afectos se manifiestan en el uso de diminutivos y palabras que pertenecen a la parte léxica del lenguaje femenino. La organización temática textual es un aspecto que se resalta en los vídeos de Viteri, y que le sirve para la construcción de un discurso de forma compartida; además de que aporta ideas basadas en su propia experiencia.

Los elementos no verbales como son la cinesia y la proxemia, están presentes en los diálogos y entrevistas de Viteri, a través de los besos en los saludos y una mayor proximidad con el entrevistador al hablar; por otro lado, los movimientos gestuales tanto de las manos y como de los brazos se realizan en un espacio más cercano al propio cuerpo, con el antebrazo casi pegado al tórax, lo que es muy común en ella, así como las piernas juntas o cruzadas por las rodillas, como parte de la representación de las características propias del lenguaje femenino.

Finalmente, las diferencias de género son un hecho fundamental de la vida humana y no es sorprendente encontrarlas reflejadas en el lenguaje. Eso cabe señalar que la distinción entre el lenguaje de hombres y mujeres es un síntoma de un problema en nuestra cultura, no el problema en sí. Básicamente refleja el hecho de que se espera que hombres y mujeres tengan diferentes intereses y roles, mantener diferentes tipos de conversaciones y reaccionar de manera diferente a otras personas. Por lo tanto, se debería prestar más atención al uso del lenguaje en el contexto social.

La igualdad legal entre hombres y mujeres y la masiva incorporación de la mujer a todos los ámbitos públicos y privados han modificado la forma de hablar, han feminizado muchos términos referidos a oficios, cargos y profesiones y han forzado cambios expresivos en el lenguaje formal y políticamente correcto. Sin embargo, basta escuchar con intención cualquier conversación cotidiana -da igual que sea banal o profesional-, para descubrir que el lenguaje habitual está lleno de expresiones y estructuras que ayudan a identificar al orador y a la intención de su discurso. 


\section{Bibliografía}

Alcaldía de Guayaquil. (2019, junio, 26). Dra. Cynthia Viteri - Radio Élite [Video]. De https://www.youtube.com/watch?v=LrpIEnRs7NY

Alcaldía de Guayaquil. (2019, agosto 26). Entrevista a la Alcaldesa Cynthia Viteri - RTS

[Video]. De https://www.youtube.com/watch?v=RXUL05XIj6s

Alcaldía de Guayaquil. (2019, agosto, 28). Dra. Cynthia Viteri - Enlace radial Radio Centro [Video]. De: https://www.youtube.com/watch?v=vPJQKA5rJMg

Álvarez, J. (2019, July 06). academialatin. Retrieved November 01, 2019, from Academia Latín: https://academialatin.com/curso/linguistica-general/pragmatica-analisisdiscurso/

Arnedo, E., Escapa, R., Valcárcel, A., Rubiales, A., Ruiz, A., Duhrkop, B., ... Escario, P. (2006). Mujeres en Red. Retrieved from http://www.mujeresenred.net: http://www.mujeresenred.net/spip.php?article536

Bengoechea, M. (1996). Intercambia Educalab. Retrieved from intercambia educalab es: http://intercambia.educalab.es/wpcontent/uploads/2016/05/MBengoetxeacomfem.pdf

Ecuavisa. (2019, octubre, 3). Cynthia Viteri critica medidas económicas del Gobierno [Video]. De https://www.youtube.com/watch?v=3DUNxbzi-oU

Horn, L. R., \& Ward. (2006). The Handbook of Pragmatics. Oxford: Blackwell Publishing.

Ishihara, N., \& Cohen, A. D. (2010). Teaching and Learning Pragmatics, Where Language and Culture Meet. Harlow: Pearson Educational Limitd.

Lakoff, R. (1975). El lenguaje y el lugar de la mujer. Barcelona: Ricou.

Lozano Domingo, I. (1995). Lenguaje femeninno, lenguaje masculino. Madrid: Minerva Ediciones.

Moreno, C. (2016). itaipue.org.mx. Retrieved from itaipue.org.mx: https://itaipue.org.mx/documentos/reflexiones/TEXTO.CECILIA_MORENO_ROME RO.pdf

Pease, A., \& Pease, B. (2001). Why Men Don't Listen \& Women Can't read Maps: How we're different and what to do about it. United Kingdom: Orion Publishing Group.

Tannen, D. (1991). You Just Don't Understand: Women and Men in Conversation. New York: Ballantine Books.

Tannen, D. (1994). Género y discurso. Barcelona: Paidós.

Teleamazonas Ecuador. (2019, marzo, 21). Las propuestas de Cynthia Viteri para la Alcaldía de Guayaquil - Teleamazonas [Video]. De

https://www.youtube.com/watch?v=Z7e4mA8XUdg

Tusón, A. (2016). Enunciación. Retrieved from revistas.udistrital.edu.co: http://revistas.udistrital.edu.co/ojs/index.php/enunc

Viteri, C. (2019, agosto, 1). ¡Con Guayaquil no se juega! [Video]. De https://www.youtube.com/watch?v=nCKEesewxfk 
Wodak, R. (1997). Introduction: some important issues in the research of gender and discourse. London: Sage.

Yaguello, M. (1978). Les mots et le femmes. Paris: Payot.

Yule, G. (2006). The Study of Language. Cambridge: Cambridge University Press. 


\section{Autores}

MIGUEL ROMÁN-JARAMILLO obtuvo su Certificado de Interprete Comunitario en la Asociación Educacional de Trabajadores en Londres (Reino Unido) en 2014. Obtuvo su Certificado de Enseñanza para Adultos en el Colegio del Noreste de Londres (programa de la Universidad de Middlesex) en 2007.

Actualmente es estudiante de octavo semestre de la Carrera de Pedagogía de los Idiomas Nacionales y Extranjeros, de la Universidad Central del Ecuador. Es representante estudiantil ante Consejo de Carrera en la Carrera de Pedagogía de los Idiomas Nacionales y Extranjeros, de la Universidad Central del Ecuador.

MAGALI ARÉVALO- ARTETA actualmente es estudiante de octavo semestre de la Carrera de Pedagogía de los Idiomas Nacionales y Extranjeros, de la Universidad Central del Ecuador. 